\title{
Manipulating Size and Shape of Silica Nanoparticles with Liquid-Phase Transmission Electron Microscopy
}

Jovana Zečević ${ }^{1}$, Krijn P. de Jong ${ }^{1}$ and Niels de Jonge e $^{2,3}$

1. Utrecht University, Inorganic Chemistry and Catalysis, Utrecht, The Netherlands.

2. INM - Leibniz Institute for New Materials, Saarbrücken, Germany.

3. Department of Physics, University of Saarland, Saarbrücken, Germany

Recently developed liquid-phase transmission electron microscopy (LP-TEM) holds great potential for dynamic imaging of relevant processes taking place in liquids at nanoscale, such as the evolution of nanoparticles during synthesis or structural changes of nanomaterials under working conditions [1]. LPTEM studies performed in the past years mainly focused on the growth of metallic nanoparticles from solutions, and pointed to important effects the electron beam can have on systems under observation [2]. Amongst various electron beam effects observed in LP-TEM, radiolysis of water and aqueous solutions leads to the formation of species such as $\mathrm{e}_{\mathrm{aq}}^{-}, \mathrm{H}^{-}, \mathrm{OH}^{-}, \mathrm{H}_{2}, \mathrm{H}_{2} \mathrm{O}_{2}, \mathrm{H}^{+}, \mathrm{OH}^{-}$. These species can locally change the chemistry in the LP-TEM cell, triggering reactions such as reduction of metal ions. In fact, electron beam induced nucleation of metal nanoparticles has been one of the main focuses of the LPTEM studies so far [2].

The goal of our work was to study the influence of the electron beam on silica nanoparticles in water. We imaged about $40 \mathrm{~nm}$-diameter silica spheres in a water environment. To prepare a sample, a droplet of sonicated silica-ethanol suspension $(\sim 0.5 \mu \mathrm{l})$ was placed on top of a Si microchip with SiN window (Protochips Inc., NC, USA), allowing the ethanol to evaporate within a minute, so that the silica spheres firmly attached to the $\mathrm{SiN}$ window. A $0.3 \mu \mathrm{l}$ droplet of water was then placed on the microchip, and the LP-TEM cell was closed by placing a second microchip on top, with its $\mathrm{SiN}$ window facing downward (Fig. 1). Having the silica spheres fixed to the SiN window allowed us to study electron beam effect on individual silica nanoparticles, as opposed to having freely moving silica particles in water, which were reported to create several hundreds of nanometers deposits on the SiN window upon scanning with a focused electron beam [3]. Imaging was performed in scanning TEM (STEM) mode at $200 \mathrm{keV}$ beam energy at different magnifications (using an ARM200F, JEOL, Japan).

Fig. 2a shows a STEM image (20 $\mu$ s dwell time) of a silica agglomerate fixed to the top SiN window. Upon longer exposure to focused scanning electron beam (50 frames, total of $\sim 5 \mathrm{~min}$ ), elongation occurred of the spherical individual silica particles, and of the silica agglomerate, in the direction of the line scanning (Fig. 2b). This observation points towards the presence of a two-fold effect of the focused electron beam on the silica particles and their environment. Firstly, the formation of $\mathrm{OH}^{-}$species upon electron beam induced water radiolysis might have led to a local increase of the $\mathrm{pH}$ inducing a local dissolution of the silica nanoparticles. Secondly, a fraction of the dissolved silica seems to have been redeposited on the sides of the silica agglomerate in horizontal direction, i.e. in the scanning direction. Deposition of silica upon electron beam irradiation in a liquid environment has been observed in the earlier study [3] but not with a directional component as observed here. To further test this effect, we changed the direction of scanning by a 90 degrees rotation, and exposed the particles from Fig. $2 \mathrm{~b}$ to another $\sim 5$ min of scanning, now with a vertical line scan direction. As can be seen in Fig. $2 c$, the elongated silica particles from Fig. $2 \mathrm{~b}$ returned to a nearly spherical shape, while the elongation of the whole agglomerate was reduced. The size of the agglomerate also seems to have been reduced. Possibly, 
dissolution and redistribution of silica species has taken place within the interparticle pores of the agglomerate, leading to the fusion of individual silica particles, a loss of pore space, and a reduction of the total volume of the agglomerate.

In conclusion, these results show that a focused electron beam can break silica bonds, and can induce the re-deposition of silica onto silica nanoparticles. The electron beam can thus reshape and resize silica nanoparticles in aqueous environment. Observed phenomena can potentially be utilized for nanoscale manipulations, and are also relevant for future LP-TEM investigations of silica based samples.

\section{References:}

[1] N. de Jonge, F. M. Ross, Nat. Nanotech. 6 (2011) p. 695.

[2] J. E. Evans et al. Nano Lett. 11 (2011) p. 2809.

[3] M. W. P. van de Put et al., Small (2014) DOI: 10.1002/smll.201400913

[4] J.Z. and K.P.dJ. acknowledge funding from the NRSCC and an ERC Advanced Grant. NdJ thanks Protochips Inc., NC, USA, for providing the LP-TEM system.

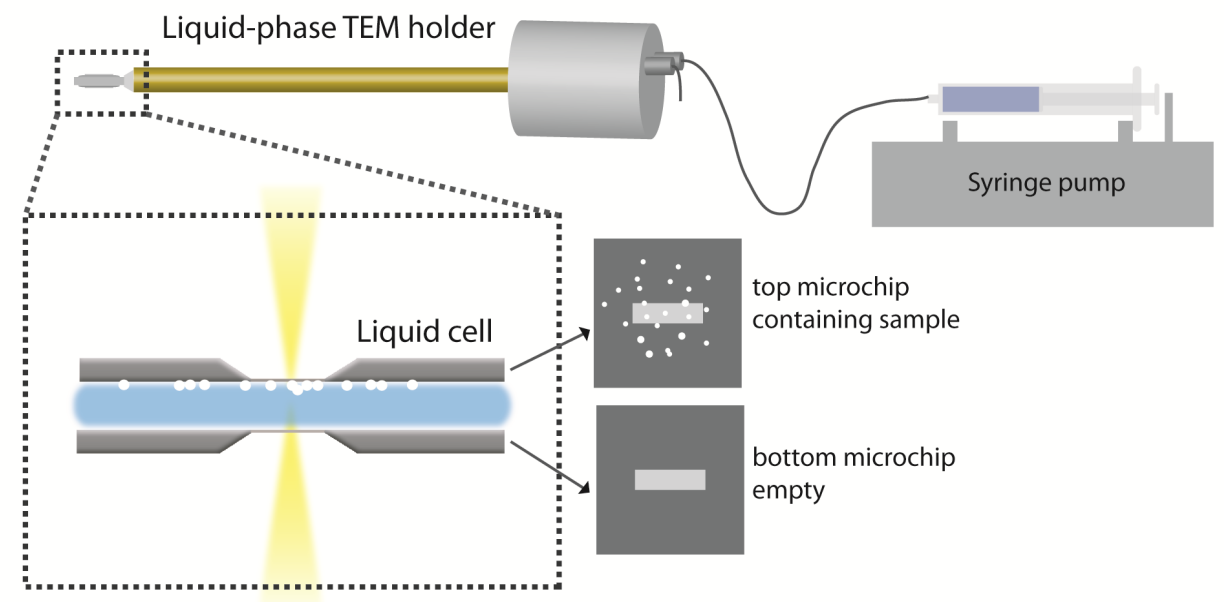

Figure 1. Schematic representation of experimental setup, showing liquid-phase TEM holder containing liquid cell composed of two $\mathrm{Si}$ microchips with $50 \mathrm{~nm}$ thin $\mathrm{SiN}$ window and water layer in between. Silica sample was deposited on the top microchip to enhance the imaging resolution.
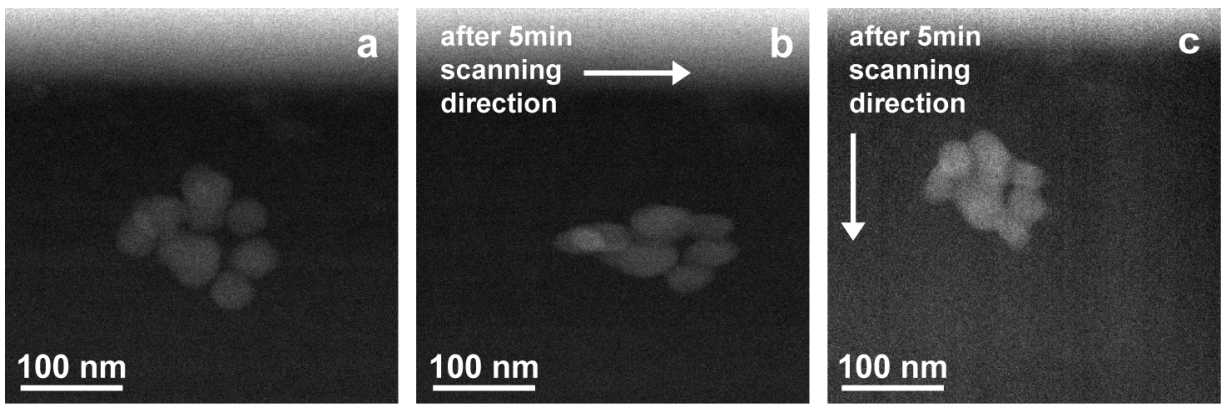

Figure 2. Electron beam induced morphological changes of agglomerate of silica particles in water, imaged with STEM. (a) STEM image of an agglomerate recorded at 600,000x magnification. (b) STEM image of silica agglomerate after 5 min scanning in horizontal direction. (c) STEM image of silica agglomerate from (b) after 5 min scanning in vertical direction. 